\title{
Alterações na estrutura da comunidade de artrópodes de solo provocadas pelo manejo
}

O solo é uma estrutura trifásica e ativa que pode servir de habitat a vários organismos que são responsáveis por diversas atividades no ambiente, envolvendo a transformação da matéria orgânica, a ciclagem e liberação de nutrientes, e a estruturação do solo. As funções desempenhadas pelos invertebrados do solo são indispensáveis para manutenção dos processos biológicos do solo, sendo inteiramente conectada à preservação da fertilidade do solo nos vários ecossistemas. Portanto, o objetivo desse trabalho foi avaliar a influência do manejo em áreas sob diferentes cultivos, hortaliças, girassol, arroz em sistema agroflorestal (SAF) e vegetação nativa, na composição e distribuição dos artrópodes de solo nos períodos seco e chuvoso em Redenção, Ceará. Para capturar os indivíduos do solo foram utilizadas armadilhas 'pitfall', que permaneceram no campo por sete dias. Após a triagem, foram calculados os índices de diversidade de Shannon-Wiener e de uniformidade de Pielou. A similaridade das áreas e de grupos foi avaliada com análise multivariada empregando técnica de componentes principais (ACP) e de análises de fatores (AF). A abundância e a diversidade dos artrópodes de solo variam em função dos manejos e com o período sazonal da região. Os grupos Entomobryomorpha e Formicidae foram os mais abundantes nas áreas nos dois períodos do estudo. A área do SAF favoreceu a comunidade de artrópodes, melhorando as condições edáficas para a manutenção da comunidade do solo.

Palavras-chave: Manejo do solo; Semiárido; Fauna edáfica.

\section{Changes in community structure of soil arthropods caused by management}

\begin{abstract}
Soil is a three-phase and active structure that can serve as a habitat for various organisms that are responsible for various activities in the environment, involving the transformation of organic matter, the cycling and release of nutrients, and the structuring of the soil. The functions performed by soil invertebrates are indispensable for maintaining the biological processes of the soil, being entirely connected to the preservation of soil fertility in the various ecosystems. Therefore, the objective of this work was to evaluate the influence of management in areas under different crops, vegetables, sunflower, rice in an agroforestry system (SAF), and native vegetation, in the composition and distribution of soil arthropods in the dry and rainy periods in Redenção, Ceará. Pitfall traps were used to capture individuals from the ground, which remained in the field for seven days. After screening, Shannon-Wiener diversity and Pielou uniformity indices were calculated. The similarity of areas and groups was assessed with multivariate analysis using the principal component (PCA) and factor analysis (PA) technique. The abundance and diversity of soil arthropods vary depending on the management and the seasonal period in the region. The groups Entomobryomorpha and Formicidae were the most abundant in the areas in the two periods of the study. The SAF area favored the arthropod community, improving soil conditions for maintaining the soil community.
\end{abstract}

Keywords: Soil management; Semi-arid; Edaphic fauna.

Topic: Ciência do Solo

Reviewed anonymously in the process of blind peer.

Cesarina Chagas de Freitas (iD)

Universidade da Integração Internacional da Lusofonia Afro-Brasileira, Brasil http://lattes.cnpq.br/5470918857181523

http://orcid.org/0000-0002-1631-3533

cesarina_chagas@hotmail.com

Isabel Cristina da Silva Araújo id

Universidade Federal do Ceará, Brasil

http://lattes.cnpq.br/1566071575904510

http://orcid.org/0000-0002-4900-1464

isabelcsa@yahoo.com.br

\section{Susana Churka Blum (iD)}

Universidade da Integração Internacional da Lusofonia Afro-Brasileira, Brasil http://lattes.cnpq.br/3470057849470057

http://orcid.org/0000-0003-2914-4780

scblum@unilab.edu.br
Received: $10 / 11 / 2020$

Approved: 15/02/2021

\author{
Olienaide Ribeiro de Oliveira Pinto (1D) \\ Universidade da Integração Internacional da Lusofonia Afro-Brasileira, Brasil \\ http://lattes.cnpq.br/8216617581480616 \\ http://orcid.org/0000-0002-8333-3665 \\ agron.olienaide@gmail.com \\ Juan Carlos Alvarado Alcócer (ib \\ Universidade da Integração Internacional da Lusofonia Afro-Brasileira, Brasil \\ http://lattes.cnpq.br/8172187725052094 \\ http://orcid.org/0000-0003-0722-7040 \\ jcalcocer@unilab.edu.br \\ Maria Ivanilda de Aguiar (iD \\ Universidade da Integração Internacional da Lusofonia Afro-Brasileira, Brasil \\ http://lattes.cnpq.br/8172187725052094 \\ http://orcid.org/0000-0002-6147-2621 \\ ivanilda@unilab.edu.br
}

\section{Referencing this:}

FREITAS, C. C.; ARAÚJO, I. C. S.; BLUM, S. C.; PINTO, O. R. O.; ALCÓCER, J. C. A.; AGUIAR, M. I.. Alterações na estrutura da comunidade de artrópodes de solo provocadas pelo manejo. Natural Resources, v.11, n.1, p.1-11, 2021. DOI: http://doi.org/10.6008/CBPC22379290.2021.001.0001

DOI: 10.6008/CBPC2237-9290.2021.001.0001 


\section{INTRODUÇÃO}

O solo é um sistema trifásico e dinâmico capaz de servir de habitat para diversos organismos, responsáveis por inúmeras funções no ambiente, incluindo a decomposição da matéria orgânica, a ciclagem e disponibilidade de nutrientes, e a estruturação do solo (BARRETTA et al., 2011; ROGER-ESTRADE et al., 2010). As atividades exercidas pelos invertebrados do solo são fundamentais para manutenção dos processos biológicos do solo, sendo inteiramente ligadas a manutenção da fertilidade do solo nos diversos ecossistemas (NUNES et al., 2008; LIMA et al., 2010; SILVA et al., 2012). Assim, pela sua relevância nos processos biológicos dos ecossistemas naturais, a fauna edáfica é utilizada como importante indicador biológico de qualidade do solo, podendo ser útil na identificação de degradação em agroecossistemas, uma vez que a diversidade da pedofauna tende a ser baixa em sistemas com elevada perturbação antrópica (BARETTA et al., 2011). Além disso, o estudo da composição, abundância e diversidade de grupos da fauna do solo é de fácil execução e baixo custo, tornando-se viável para avaliar o impacto das intervenções antrópicas nos ecossistemas.

As práticas de manejo empregadas em sistemas agrícolas interferem diretamente na condição destes indivíduos que estão exercendo atividades no ambiente (ALVES et al., 2008). Desta forma, é necessário que se faça um constante monitoramento da fauna do solo que permitirá uma indicação simples da ecologia dos agroecossistemas, uma vez que esse é modificado devido o manejo, promovendo alterações nas condições físicas e químicas do solo, como também na diversidade e abundância da pedofauna.

Estudos ressaltam que em solos agrícolas, a fauna edáfica varia de acordo com conteúdo da matéria orgânica, proteção do solo, espécies cultivadas, microclima e umidade (MANHAES et al., 2012). No semiárido, observa-se que a grande maioria das práticas agrícolas, incluindo desmatamentos e queimadas, causa redução significativa na abundância e na diversidade da fauna quando comparados com áreas sob vegetação nativa (ARAÚJO et al., 2018; LIMA et al., 2010). Por outro lado, pensando-se em evitar problemas de degradação dos recursos naturais, atualmente, tem-se adotado práticas que buscam inibir efeitos negativos causados pelo uso inadequado do solo, melhorando a produção agrícola, preservando a biodiversidade nos agroecossistemas e conservando os recursos naturais. Dentre estas práticas pode-se citar o manejo agroecológico e os sistemas agroflorestais.

Os sistemas agroflorestais (SAF) são sistemas de uso da terra em que plantas de espécies agrícolas são combinadas com espécies arbóreas sobre a mesma unidade de manejo da terra. Esta combinação tem sido uma alternativa para a melhoria nas propriedades físico-químicas de solos degradados, e também nas atividades de micro-organismos (LOURENTE et al., 2011).

Os sistemas agroflorestais proporcionam benefícios ambientais, como a conservação da biodiversidade, o sequestro de carbono e a melhoria no controle de qualidade da água, melhorando, também, a produção dos sistemas agrícolas (NAIR, 2008; WASTOWSKI et al., 2010; LIMA et al., 2011). Neste contexto, espera-se que o manejo agroflorestal favoreça a pedofauna, tornando-a mais diversa. Assim, o objetivo deste estudo foi avaliar a influência do manejo do solo na composição e distribuição de artrópodes do solo durante os dois períodos sazonais no município de Redenção, no estado do Ceará, Brasil, sob 
diferentes manejos agroecológicos.

\section{MATERIAIS E MÉTODOS}

O estudo foi realizado na Fazenda Experimental Piroás (FEP) pertencente à Universidade da Integração Internacional da Lusofonia Afro-Brasileira (UNILAB), em Redenção, Ceará. A temperatura média anual do município de Redenção varia de $26^{\circ} \mathrm{C}$ a $28^{\circ} \mathrm{C}$ e a pluviosidade anual média é de $1.062,0 \mathrm{~mm}$, com estação chuvosa de janeiro a abril (IPECE, 2014). Porém, na FEP, a média dos últimos quatro anos (20142017) foi de 1.222,0 mm anuais. Na figura 1 observa-se a precipitação mensal ocorrida nestes anos.

O clima da região é predominantemente tropical, com vegetação do tipo caatinga arbustiva densa e floresta subcaducifólia tropical pluvial (IPECE, 2015), com solos do tipo Planossolo Solódico e Podzólico Vermelho-Amarelo Eutrófico.

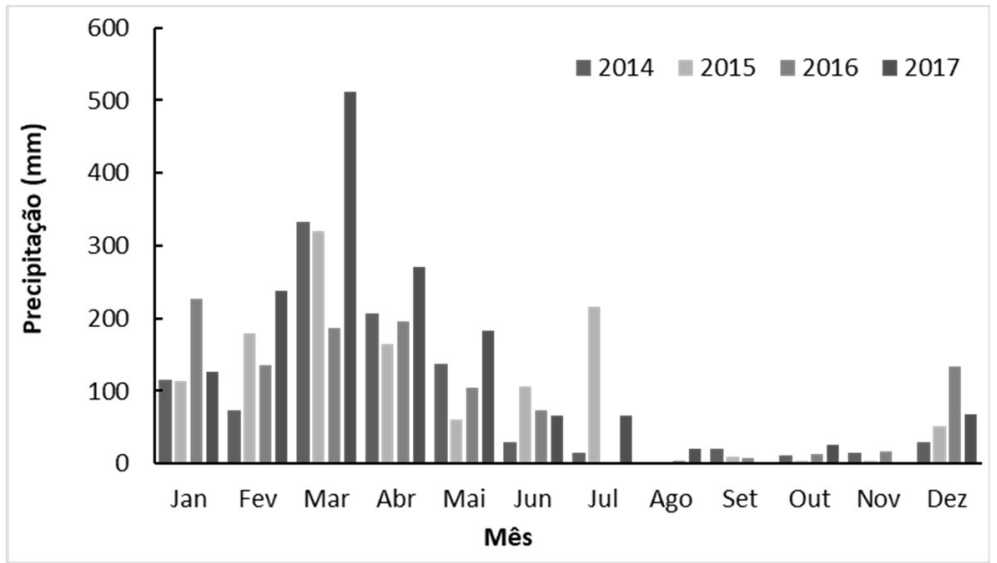

Figura 1: Precipitação pluviométrica mensal dos anos de 2014 a 2017 na Fazenda Experimental Piroás, Redenção, Ceará.

Foram avaliadas quatros áreas sob manejos distintos, sendo uma área de cultivo de hortaliças $(\mathrm{CH})$, um cultivo orgânico de girassol (CG), uma área sob sistema agroflorestal (SAF) e uma área sob vegetação nativa de caatinga (VN).

A área sob $\mathrm{CH}$, possui aproximadamente $340 \mathrm{~m}^{2}$, constituída de uma área didática, utilizadas em aulas práticas de disciplinas do curso de Agronomia da UNILAB. Assim, nesta área são realizados cultivos de diversas plantas, tais como alface, cebola, coentro, feijão guandu, várias espécies de capim, cana-de-açúcar, palma forrageira e plantas ornamentais. Utiliza-se irrigação por microaspersão, adubação orgânica (húmus de minhoca) e cobertura morta.

A área CG possui $889 \mathrm{~m}^{2}$ que teve sua vegetação nativa (secundária), composta de diversas espécies de caatinga desmatada no final do período seco de 2015. O cultivo de girassol foi realizado no período de janeiro a abril de 2016, sendo a coleta realizada no final do cultivo de girassol. Para o cultivo de girassol utilizou-se adubação a base de cama-de-frango e casca de arroz carbonizada e irrigação diária por microaspersão.

A área VN, com formação secundária (aproximadamente sete anos de pousio), com $8.100 \mathrm{~m}^{2}$, na qual se observa presença das seguintes espécies arbóreas/arbustivas Albizia polycephala (Benth.) Killip, Triplaris 
americana, Solanum paniculatum, Simarouba amara Aubl, Rhamnidium elaeocarpum Reissek, Piptadenia stipulacea (Benth.) Ducke, Mimosa tenuiflora (Willd.) Poir, Mimosa caesalpiniifolia Benth, Mimosa arenosa (Willd.) Poir, Licania rigida Benth, Jatropha sp, Guazuma ulmifolia Lam, Delonix regia, Alchornea sidifolia Mull. Arg, Bauhinia cheilantha (Bong.) Steud, Capparis cynophallophora L, Croton sonderianus Mull. Arg e Cyperus rotundus $\mathrm{L}$.

Área de SAF, era composta por vegetação nativa da caatinga que foi raleada no final do período seco de 2016, preservando-se cerca de 10\% da cobertura arbórea nativa. O material proveniente do raleamento da vegetação nativa foi depositado no solo, formando leiras no sentido contrário ao declive predominante. Realizou-se cultivo de milho e arroz, durante o período chuvoso de 2017 , sem adição de adubos e sem uso de irrigação.

As amostragens para coleta da meso e macrofauna do solo foram realizadas em novembro de 2016 (período seco) e abril de 2017 (período chuvoso), por meio da instalação de quatro armadilhas de queda (pitfall-traps) por área, espaçadas entre si em $10 \mathrm{~m}$, sendo dois no sentido norte sul e dois no sentido leste oeste, totalizando 16 armadilhas (Figura 2).

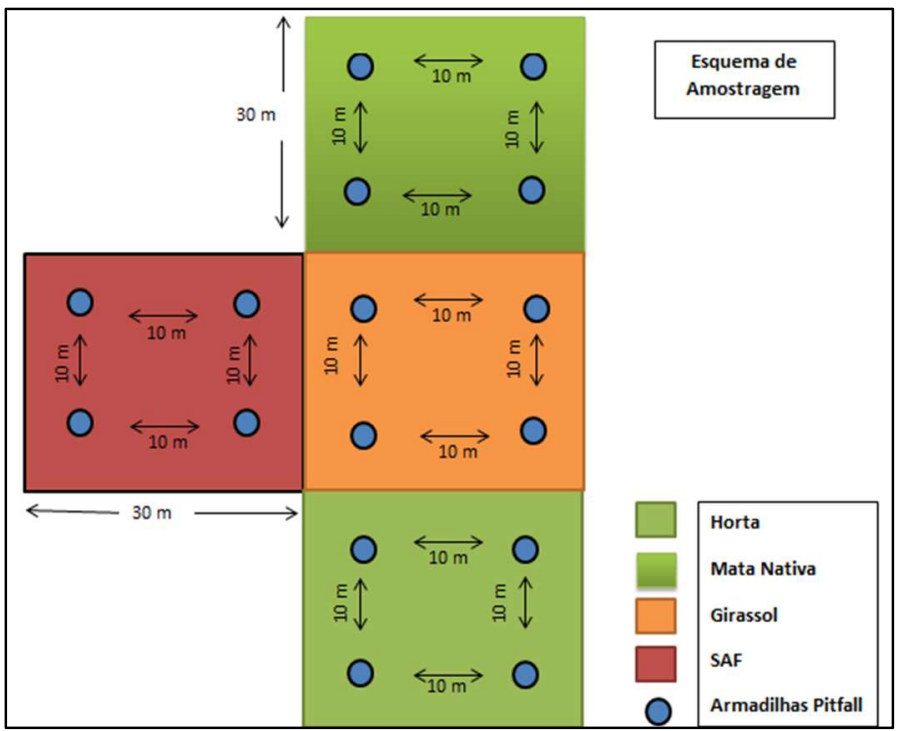

Figura 2: Esquema amostral de área onde foram instaladas as armadilhas para captura da fauna edáfica na Fazenda Experimental Piroás, Redenção, Ceará.

As armadilhas (recipiente plástico, com capacidade para $500 \mathrm{ml}$, enterradas no solo, com abertura ao nível da superfície) foram preenchidas com líquido conservante (álcool 53\% e gotas de detergente, para quebrar a tensão superficial da água) até $1 / 3$ da sua capacidade. Após a instalação, as armadilhas permaneceram no campo por 7 dias e seu conteúdo foi revisado a cada dois dias para repor o líquido conservante quando ocorria elevada evaporação, ou para evitar que transbordassem, no caso do período chuvoso.

Posteriormente, as armadilhas foram levadas para o laboratório e seu conteúdo foi limpo e transferido para solução de álcool $70 \%$. Sendo feita, na sequência, a triagem e identificação dos grupos. A identificação dos indivíduos presentes nas amostras foi realizada vertendo o conteúdo dos frascos em placa de petri e observando os organismos sob microscópio estereoscópio. O reconhecimento dos grupos 
taxonômicos foi realizado através de consulta a materiais diversos. Após identificados os indivíduos foram contados e classificados em grandes grupos taxonômicos.

A partir dos resultados obtidos, foram calculados: densidade dos grupos em número de indivíduos por armadilha por dia (AQUINO et al., 2008), riqueza da fauna (número de grupos identificados por área), diversidade e uniformidade. A diversidade dos grupos da meso e macrofauna do solo, que expressa à relação entre o número de grupos (riqueza de grupos) e a distribuição do número de indivíduos, ou seja, a quantidade entre os grupos (uniformidade ou equabilidade) foi estimada utilizando o Índice de Diversidade de ShannonWeaver ( $H^{\prime}$ ) e a uniformidade dos grupos de acordo com o Índice de Uniformidade de Pielou (e). O Índice de Diversidade de Shannon-Weaver (H), é apropriado para o uso em ecologia do solo (NUNES et al., 2008), pois leva em consideração a riqueza das espécies e sua abundância relativa, conforme a equação 1:

$$
\mathrm{H}=-\Sigma \mathrm{pi} . \log \mathrm{i} \text {. }
$$

Em que: $\mathrm{pi}=\frac{\mathrm{ni}}{\mathrm{N}} ; \mathrm{ni}=$ valor de importância de cada espécie ou grupo; e $\mathrm{N}=$ total dos valores de importância. O Índice de Uniformidade de Pielou (e), conforme MAGURRAN (2004) é um índice em que a uniformidade se refere ao padrão de distribuição dos indivíduos entre as espécies, sendo calculado de acordo com equação 2 :

$$
\mathrm{e}=\frac{\mathrm{H}}{\log }
$$

Em que: $\mathrm{H}$ = Índice de Shannon; e $\mathrm{S}=$ Número total de espécies ou grupos na comunidade. Nas áreas de estudo também foram coletadas amostras deformadas de solo, na camada de 0 a 10 $\mathrm{cm}$, para determinação do $\mathrm{pH}$, potássio $(\mathrm{K})$, fósforo $(\mathrm{P})$, sódio $(\mathrm{Na})$ e carbono orgânico total (COT), conforme metodologias descritas por (DONAGEMA et al., 2011).

As diferentes áreas, bem como os diferentes períodos de amostragens foram comparadas mediante a utilização da densidade (número de indivíduos por armadilha por dia de coleta), da riqueza total (número de grupos taxonômicos nas diferentes áreas) e da riqueza média (número médio de grupos taxonômicos presentes em cada ponto amostral). Realizou-se análise multivariada, empregando-se técnicas de análise fatorial (FA) e de componentes principais (PCA), utilizando as variáveis do solo (P, K, Na e COT) como variáveis suplementares.

\section{RESULTADOS E DISCUSSÃO}

No total, foram coletados 8482 indivíduos, distribuídos em 24 grupos taxonômicos. Os grupos identificados foram classificados em ordens, subordem, classe e família, sendo todos considerados grupos de artrópodes do solo. As ordens observadas foram Acari, Araneae, Archaeognata, Coleóptera e Larva de Coleóptera, Dermaptera, Díptera, Entomobryomorpha, Hymenoptera, Isopoda, Isoptera, Orthoptera, Poduromorpha, Pseudoscorpionida, Psocoptera, Symphypleona, Embioptera, Escorpionida e as subordens foram, Auchenorryncha, Heteroptera, Sternorryncha, Colembola. Observou-se também a como classe Diplopoda e a família Formicidae.

Durante os períodos de coleta, a abundância geral dos grupos variou principalmente no período seco (Figura 3), sendo o CG o mais abundante em relação às áreas SAF e VN. Já no período chuvoso, as áreas CG, 
CH e SAF apresentaram abundâncias semelhantes e superiores a VN. Observa-se que a vegetação nativa apresenta menor abundância nos dois períodos, ressaltando assim a importância do uso de práticas agrícolas para diversificação dos nichos ecológicos. Estudos realizados no município de Esperantina, Piauí, ressaltam que os sistemas agroflorestais, quando comparados a cultivos homogêneos, propiciam melhores características químicas do solo, bem como o aumento na abundância e riqueza de espécies da macrofauna edáfica (LIMA et al., 2010).

Durante o período seco, foi observado maior número de indivíduos, porém isso ocorreu devido à maior abundância do grupo Entomobryomorpha neste período. A área sob mata nativa foi a que apresentou menor variação nos resultados, com valores semelhantes para os períodos estudados (Figura 3).

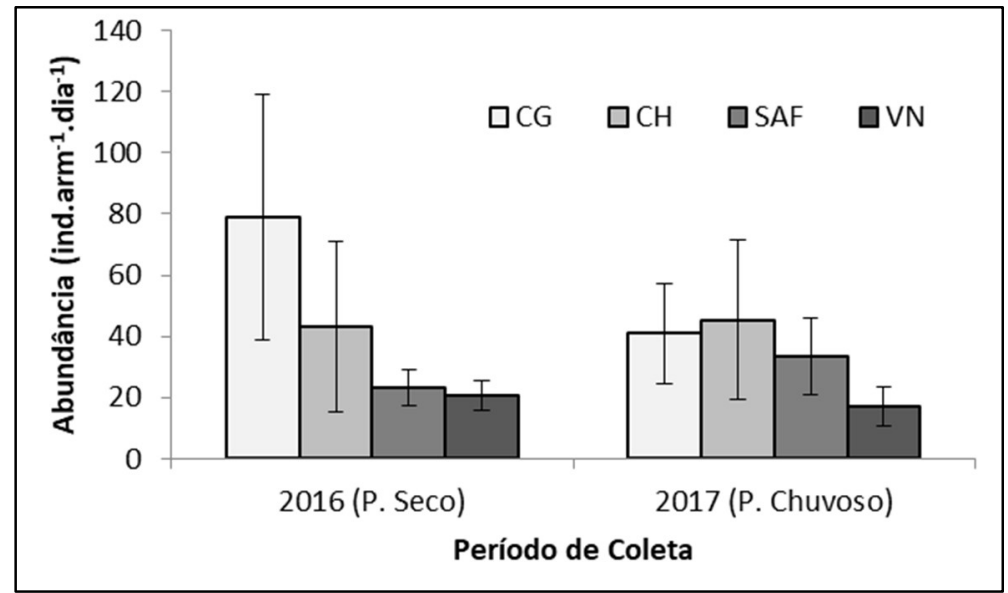

Figura 3: Abundância de artrópodes do solo coletados em diferentes áreas durante dois períodos seco e chuvoso em Redenção, Ceará. CH: cultivo orgânico de hortaliças: CG: cultivo orgânico de girassol; SAF: sistema agroflorestal: VN: vegetação nativa de caatinga.

No período seco, obteve-se alta abundância de colêmbolos, que foi constatada em estudo realizado na pastagem impactada pela queimada (BARETTA et al., 2011), o que pode indicar degradação. A retirada das árvores e a redução do aporte de resíduos pela queima afetam o microclima, os nichos e a quantidade e qualidade de recursos para alimentação e nidificação da pedofauna (LIMA et al., 2010) fatores limitantes que podem reduzir a abundância da pedofauna.

Verificou-se que os grupos Entomobryomorpha e Formicidae apresentaram o maior percentual de indivíduos, alternando-se em predomínio em cada sistema e nos dois períodos de amostragem (Tabela 1). Nas áreas cultivadas com girassol e com hortaliças, constatou-se que o grupo Entomobryomorpha apresentou maior percentual de indivíduos no período seco, enquanto que no período chuvoso, o maior percentual foi de Formicidae.

O grupo Formicidae apresenta grande diversidade nos hábitos alimentares, podendo ser predadoras ou consumidoras primarias, colaborando, assim, na ciclagem de nutrientes (VASCONCELOS, 2008). Esse grupo possui um enorme potencial biótico, formando muitas populações, porém algumas espécies desse grupo podem causar possíveis danos econômicos por alguns motivos, como por exemplo, cortando as folhagens das plantações (KORASAKI et al., 2013). Nos trabalhos de Vargas et al. (2013) o grupo Formicidae foi o mais abundante nos três sistemas estudados, portanto esse comportamento é comum, elas são capazes 
de desempenhar importantes papéis em relação aos processos ecológicos, e ajudam também na dispersão de sementes, na estrutura química e física do solo e na ciclagem de nutrientes (VASCONCELOS, 2008).

Tabela 1: Abundância de artrópodes do solo (ind. $\mathrm{Arm}^{-1} . \mathrm{dia}^{-1}$ ) nas áreas em estudo na Fazenda Experimental Piroàs, Redenção, Ceará.

\begin{tabular}{|c|c|c|c|c|}
\hline \multirow[t]{3}{*}{ Grupos } & \multicolumn{3}{|c|}{ Áreas de manejo } & \multirow[b]{2}{*}{ VN } \\
\hline & CG & $\mathrm{CH}$ & SAF & \\
\hline & & \multicolumn{2}{|l|}{ Período Seco } & \\
\hline Acari & $1,3 \pm 0,65$ & $1,32 \pm 0,87$ & $3,96 \pm 3,28$ & $1,96 \pm 2,10$ \\
\hline Araneae & $0,32 \pm 0,46$ & $0,61 \pm 0,27$ & $0,64 \pm 0,63$ & $0,54 \pm 0,36$ \\
\hline Coleoptera & $0,61 \pm 0,67$ & $0,71 \pm 0,65$ & $0,29 \pm 0,12$ & $0,32 \pm 0,47$ \\
\hline Díptera & $1,11 \pm 0,98$ & $10,39 \pm 15,60$ & $1,89 \pm 0,78$ & $0,93 \pm 0,81$ \\
\hline Entomobryomorpha & $58,18 \pm 45,72$ & $12,14 \pm 14,67$ & $8,89 \pm 3,61$ & $5,86 \pm 3,53$ \\
\hline Formicidae & $13,18 \pm 12,21$ & $5,50 \pm 2,36$ & $6,32 \pm 2,35$ & $10,0 \pm 7,47$ \\
\hline Orthoptera & $0,04 \pm 0,07$ & $0,36 \pm 0,25$ & $0,25 \pm 0,14$ & $0,25 \pm 0,24$ \\
\hline Poduromorpha & $2,04 \pm 1,56$ & $3,04 \pm 4,51$ & $0,46 \pm 0,54$ & $0,14 \pm 0,29$ \\
\hline \multirow[t]{2}{*}{ Outros } & $2,32 \pm 1,46$ & $8,96 \pm 14,22$ & $0,57 \pm 0,48$ & $0,57 \pm 0,51$ \\
\hline & & \multicolumn{2}{|c|}{ Período Chuvoso } & \\
\hline Acari & $0,71 \pm 0,42$ & $4,64 \pm 2,78$ & $0,71 \pm 0,95$ & $0,82 \pm 0,60$ \\
\hline Araneae & $0,89 \pm 0,61$ & $1,61 \pm 1,02$ & $1,00 \pm 0,31$ & $0,71 \pm 0,61$ \\
\hline Coleoptera & $2,11 \pm 2,39$ & $2,86 \pm 0,52$ & $5,25 \pm 1,11$ & $3,64 \pm 1,01$ \\
\hline Díptera & $2,79 \pm 1,35$ & $3,11 \pm 3,63$ & $1,64 \pm 0,70$ & $1,86 \pm 0,75$ \\
\hline Entomobryomorpha & $12,29 \pm 3,44$ & $5,21 \pm 5,30$ & $16,71 \pm 5,58$ & $5,00 \pm 4,34$ \\
\hline Formicidae & $6,46 \pm 6,87$ & $15,75 \pm 15,34$ & $4,04 \pm 5,62$ & $3,57 \pm 0,97$ \\
\hline Orthoptera & $1,11 \pm 1,08$ & $1,50 \pm 1,38$ & $0,21 \pm 0,14$ & $0,21 \pm 0,25$ \\
\hline Poduromorpha & $3,04 \pm 4,18$ & $3,00 \pm 2,89$ & $0,82 \pm 1,11$ & $0,11 \pm 0,14$ \\
\hline Outros & $1,54 \pm 0,82$ & $8,96 \pm 8,28$ & $2,82 \pm 1,95$ & $1,57 \pm 0,31$ \\
\hline
\end{tabular}

CH: cultivo orgânico de hortaliças: CG: cultivo orgânico de girassol; SAF: sistema agroflorestal: VN: vegetação nativa de caatinga.

Diferenças significativas foram observadas na diversidade de artrópodes do solo para as comparações entre as áreas e entre os períodos de coleta, sendo a maior diversidade estimada para a área do SAF no período seco, enquanto a menor foi na área de cultivo de girassol, esse menor valor está relacionado principalmente na cobertura do solo e sombreamento da área, com o solo desprotegido para o plantio, mesmo sendo agroecológico, pode atingir de formas negativas em diversos ninchos naquele sistema (Tabela 2). No período chuvoso, a maior diversidade foi observada na área de horta, porém os valores não diferem tanto, relatando então, que as áreas são consideradas com boa diversidade ecológica.

O maior percentual de indivíduos encontrados durante a estação seca na área cultivada com girassol contribuiu para a redução do índice de diversidade durante esse período (Tabela 02), pois quanto maior a densidade da fauna, maior a chance de um grupo predominar, como foi o caso do grupo Entomobryomorpha, que representou $58,18 \pm 45,72 \%$ do total. Essas variáveis também influenciaram a equabilidade, reduzindo-a, quando há predomînio de poucos grupos em termos de número de indivíduos.

Tabela 2: Valores do índice de diversidade e índice de uniformidade para cada área sob avaliação e durante os dois períodos de coleta, em Redenção, Ceará.

\begin{tabular}{|c|c|c|c|c|}
\hline \multirow[t]{2}{*}{ Índices } & \multicolumn{3}{|c|}{ Manejo } & \multirow[b]{2}{*}{$\mathrm{VN}$} \\
\hline & $\mathrm{CH}$ & $\mathrm{CG}$ & SAF & \\
\hline & & \multicolumn{2}{|c|}{ Período Seco } & \\
\hline Shannon & 0.99 & 1,39 & 1,50 & 1,30 \\
\hline \multirow[t]{2}{*}{ Pielou } & 0,43 & 0,58 & 0,66 & 0,59 \\
\hline & & \multicolumn{2}{|c|}{ Período Chuvoso } & \\
\hline Shannon & 1,19 & 1,38 & 1,01 & 1,23 \\
\hline Pielou & 0,49 & 0,55 & 0,39 & 0,54 \\
\hline
\end{tabular}

CH: cultivo orgânico de hortaliças: CG: cultivo orgânico de girassol; SAF: sistema agroflorestal: VN: vegetação nativa de caatinga.

Comparando as áreas de horta e vegetação nativa, observa-se pequenas variações no índice de 
diversidade $\left(\mathrm{H}^{\prime}\right)$ e índice de uniformidade (e) nos dois períodos, mostrando então a agrícultura com valores ecológicos semelhantes a vegetação nativa. Valores para o índice de diversidade e para o índice de uniformidade na área de vegetação nativa durante o período chuvoso (Tabela 3) são diferentes de outros estudos de fauna edáfica que foram realizados em solos do bioma Caatinga (Floresta Tropical Seca), que demonstraram altos valores de riqueza, diversidade e uniformidade nos períodos chuvosos (NUNES et al., 2009).

Observou-se que o pH variou de ácido, com menor valor sendo observado no $\operatorname{SAF}(5,5)$ a levemente alcalino em $\mathrm{CH}$, com valor de 7,2 (Tabela 3). O fósforo não foi detectado nos tratamentos de cultivo de hortaliças e SAF, porém seu teor no manejo de girassol foi elevado, diferindo significativamente entre os solos do sistema de vegetação nativa. Nos solos do SAF e VN, os valores de $\mathrm{pH}, \mathrm{Na}, \mathrm{K}$ e COT foram semelhantes, isso ocorre devido as áreas serem próximas e também o sistema agroflorestal ser novo.

Tabela 3: Características químicas do solo, analisadas para no período seco a uma profundidade de $0-10 \mathrm{~cm}$ nas áreas de diferentes manejos, Redenção, Ceará.

\begin{tabular}{|c|c|c|c|c|c|}
\hline Área & $\begin{array}{l}\mathrm{pH} \\
\mathrm{H}_{2} \mathrm{O}\end{array}$ & $\begin{array}{l}\mathrm{P} \\
\mathrm{mg} \mathrm{kg}^{-1}\end{array}$ & $\begin{array}{l}\mathrm{K} \\
\mathrm{Cmol}_{\mathrm{c}} \cdot \mathrm{kg}^{-1}\end{array}$ & $\begin{array}{l}\mathrm{Na} \\
\mathrm{Cmol}_{\mathrm{c}} \cdot \mathrm{kg}^{-1}\end{array}$ & $\begin{array}{l}\text { COT } \\
\text { Dag. kg-1 }\end{array}$ \\
\hline $\mathrm{CH}$ & 7,2 & ND & $0,04 \pm 0,01$ & $0,11 \pm 0,07$ & $0,94 \pm 0,47$ \\
\hline CG & 6,1 & $11,25 \pm 4,49$ & $0,11 \pm 0,06$ & $0,07 \pm 0,06$ & $1,89 \pm 0,32$ \\
\hline SAF & 5,4 & ND & $0,07 \pm 0,01$ & $0,03 \pm 0,00$ & $1,65 \pm 0,70$ \\
\hline VN & 5,5 & $3,46 \pm 7,74$ & $0,13 \pm 0,06$ & $0,03 \pm 0,01$ & $1,84 \pm 0,89$ \\
\hline
\end{tabular}

O aumento dos valores de nas áreas de CH e CG devem-se, provavelmente, as condições do solo, onde há abertura do terreno para uso agrícola para o uso de uma só cultura, deixando o solo sem cobertura e desprotegido. Os valores de P e K no CG e VN foram maiores no CG. Segundo Brady et al. (2013) o P e o K são exigidos em grandes quantidades pelas plantas, pois auxiliam no processo de fotossíntese e, além disso, o K tem papel importante na absorção de água pelas raízes das plantas.

As dissimilaridades entre as áreas na composição e distribuição dos artrópodes do solo, que demonstram o efeito das alterações decorrentes das práticas de manejo e uso do solo, foram confirmadas pelas correlações entre as variáveis e fatores obtidos no componente principal e análise fatorial. Avaliando as correlações entre a varíaveis, foram obtidos sete fatores que apresentaram correlação significativa ( $p$ $<0,05)$ com as variáveis originais, onde se pode verificar quais fatores pertencem às variáveis e quanto de cada componente é explicado por cada variável (Tabela 4).

$\mathrm{Na}$ análise fatorial foram formados sete fatores, sendo que os dois primeiros representaram mais de $59 \%$ da variação (Figura 4). As variáveis com maior contribuição no primeiro fator foram Araneae, Orthoptera e Outros, enquanto no segundo foram Coleoptera, Formicidae e Poduromorpha. No terceiro fator, Diptera e Formicidae fizeram a maior contribuição. No quarto fator foram Acari e Entomobryomorpha (Tabela 4).

Quanto à análise multivariada, nota-se que os grupos Formicidae, Acari, Poduromorpha, Orthoptera (Figura 4) sofrem uma maior influência das variáveis de Na e P, pois se encontram no mesmo quadrante do gráfico. Nesse tipo de gráfico, quanto mais próximo da extremidade do círculo, mais uma variável é significativa para a análise. Nesse sentido, o grupo Araneae tem uma maior significância que Coleoptera e a riquezas de grupos mais que o Entomobryomorpha. O COT teve uma maior influência sobre grupos como 
Araneae e Coleoptera. Percebe-se também que a área de vegetação nativa teve comportamento semelhante nos dois períodos (seco e chuvoso). Assim como o $\mathrm{CH}$ seco foi semelhante ao CG chuvoso.

Tabela 4: Correlações entre as variáveis originais (Artrópodes e Índices) e os fatores ecológicos.

\begin{tabular}{lllll}
\hline Variáveis & Fator 1 & Fator 2 & Fator 3 & Fator 4 \\
\hline Aca & $-0,39$ & 0,50 & $-0,15$ & $-0,62$ \\
Ara & $-0,91$ & $-0,09$ & $-0,32$ & $-0,17$ \\
Col & $-0,42$ & $-0,77$ & -12 \\
Dip & $-0,35$ & 0,18 & $-0,25$ & 0,02 \\
Ent & 0,39 & 0,19 & 0,81 & 0,67 \\
For & $-0,34$ & 0,63 & 0,18 & 0,46 \\
Ort & $-0,83$ & 0,35 & $-0,47$ & 0,06 \\
Pod & $-0,61$ & 0,51 & $-0,39$ & 0,52 \\
Out & $-0,89$ & 0,14 & 0,24 & 0,00 \\
H & $-0,49$ & $-0,69$ & 0,37 & 0,03 \\
E & $-0,40$ & $-0,85$ & 0,42 & 0,09 \\
S & 0,07 & 0,68 & 0,18 & $-0,33$ \\
\hline Aca: Acari, Ara: Arani
\end{tabular}

Aca: Acari, Ara: Araneae; Col: Coleoptera; Dip: Diptera; Ent: Entomobryomorpha; For: Formicidae; Ort: Orthoptera; Pod: Poduromorpha; Out: Outros; H: ìndice de diversidade de Shannon; E: índice de equabilidade de Pielou; S: riqueza de espécies.

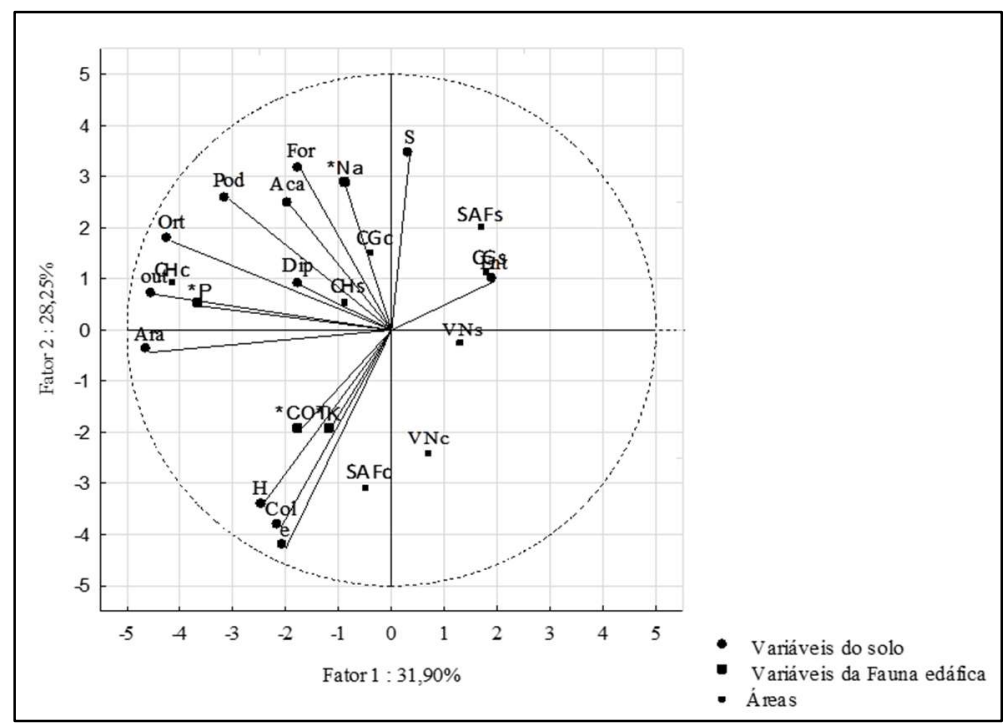

Figura 4: Círculo de correlação entre as variáveis originais (artrópodes e índices do solo) e os fatores da análise de componentes principais para as áreas cultivadas girassol (CGc e CGs), horta (CHc e CHs), sistema agroflorestal (SAFc e SAFs) e vegetação nativa (VNc e VNs) nos períodos secos (s) e chuvoso (c), em Redenção, Ceará. Aca: Acari, Ara: Araneae; Col: Coleoptera; Dip: Diptera; Ent: Entomobryomorpha; For: Formicidae; Ort: Orthoptera; Pod: Poduromorpha; Out: Outros; H: indice de diversidade de Shannon; E: índice de equabilidade de Pielou; S: riqueza de espécies.

Em termos biológicos, as correlações positivas podem significar uma relação direta entre os grupos, mas devem ser analisadas em maior detalhe em estudos futuros. A constatação de que os artrópodes do solo são influenciados pelas condições ambientais tem sido confirmada por diversos estudos (AQUINO et al., 2008; BARETTA et al., 2011; FERNANDES et al., 2011; MOÇO et al., 2010), Novos estudos, utilizando outros métodos de coleta, são necessários para determinar com maior precisão os padrões de distribuição e a diversidade de espécies de artrópodes do solo nesse ambiente.

\section{CONCLUSÕES}

A abundância e diversidade de artrópodes do solo nos sistemas de produção variam dependendo do manejo e do período na região. Os grupos Entomobryomorpha e Formicidae foram os mais abundantes em 
cada área e durante os dois períodos do estudo. A área de sistema agroflorestal no período seco apresentou maior diversidade e uniformidade na comunidade de artrópodes no solo.

O grupo Entomobryomorpha foi o mais abundante nos sistemas estudados e no GR (período seco) houve uma maior representatividade, isto pode estar relacionado a uma maior disponibilidade de alimento e os restos culturais depositados na área, como também pela não homogeneidade. Os valores de abundância total não diferiram nas áreas de $\mathrm{CH}$ e VN em relação aos seus períodos avaliados.

\section{REFERÊNCIAS}

ALVES, M.; SANTOS, J. C. P.; GOIS, D. T.; ALBERTON, J. V.; BARETTA, D.. Macrofauna do solo influenciada pelo uso de fertilizantes químicos e dejetos de suínos no oeste do estado de Santa Catarina. Revista Brasileira de Ciência do Solo, v.32, n.2, p.589-598, 2008. DOI: https://doi.org/10.1590/S0100-06832008000200014

AQUINO, A. M.; SILVA, R. F.; MERCANTE, F. M.; CORREIRA, M. E. F.; FATIMA, M.; LAVELLE, P.. Invertebrate soil macrofauna under different ground cover plants in the notill system in the Cerrado. European Journal of Soil Biology, v.44, p.191-197, 2008. DOI:

http://doi.org/10.1016/j.ejsobi.2007.05.001

ARAÚJO FILHO, J. A.; BARBOSA, T. M. L.. Sistemas agrícolas sustentáveis para regiões semiáridas. Sobral: EmbrapaCaprinos, 2018

BARETTA, D.; SANTOS, J.; SEGAT, J. C.; GEREMIA, E. V.; OLIVEIRA FILHO, L. C. I.; ALVES, M. V.. Fauna edáfica e qualidade do solo. In: Tópicos Ciência do Solo. 7 ed. 2011. p.119-170.

BRADY, N. C.; WEIL, R. R.. Elementos da natureza e propriedades dos solos. 3 ed. Porto Alegre: Bookman, 2013.

DONAGEMA, G. K.; CAMPOS, D. V. B.; CALDERANO, S. B.; TEIXEIRA, W. G.; VIANA, J. H. M.. Manual de métodos de análise de solo. 3 ed. Brasília: Embrapa, 2011.

FERNANDES, M. M.; MAGALHÃES, L. M. S.; PEREIRA, M. G.; CORREIA, M. E. F.; BRITO, R. J.. Influência de diferentes coberturas florestais na fauna do solo na 34 Flora Mário Xavier, no município de Seropédica, RJ. Floresta, v.41, p.533540, 2011. DOI: http://doi.org/10.5380/rf.v41i3.24045

IPECE. Instituto de Pesquisa e Estratégia Econômica do Ceará. Perfil Básico do Município, Redenção. Fortaleza: IPECE, 2014.

IPECE. Instituto de Pesquisa e Estratégia Econômica do Ceará. Perfil Básico Municipal, Redenção. Fortaleza: SEPLAG, 2015.

KORASAKI, V.; MORAIS, J. W.; BRAGA, R. F.. Macrofauna. In: MOREIRA, F. M. S.; CARES, J.; ZANETTI, R.; STURMER, S.. 0 ecossistema solo: Componentes, relações ecológicas e efeitos na produção vegetal. Lavras: UFLA, 2013. p.121-200.

LIMA, S. S.: AQUINO, A. M.; LEITE, L.F. C.; VELÁSQUEZ, E.; LAVELLE, P. Relação entre macrofauna edáfica e atributos químicos do solo em diferentes agroecossistemas. Pesquisa Agropecuária Brasileira, v.45, n.03, 2010. DOI:

\section{https://doi.org/10.1590/S0100-204X2010000300013}

LIMA, S. S.; LEITE, L. F. C.; OLIVEIRA, F. C.; COSTA, D. B.. Atributos químicos e estoques de carbono e nitrogênio em Argissolo Vermelho-Amarelo sob sistemas agroflorestais e agricultura de corte e queima no norte do Piauí. Revista Árvore, v.35, p.51-60, 2011.

LOURENTE, E. R. P.; MERCANTE, F. M.; ALOVISI, A. M. T.; GASPARINI, A. S.; GOMES, C. F.; NUNES, C. M.. Atributos microbiológicos, químicos e físicos do solo sob diferentes sistemas de manejo e condições de Cerrado. Pesquisa Agropecuária Tropical, v.41, p.20-28, 2011.

MANHAES, C. M. C.; FRANCELINO, F. M. A.. Estudo da interrelação da qualidade do solo e da serapilheira com a fauna edáfica utilizando análise multivariada. Nucleus, v.9, n.2, 2012.

MOÇO, M. K. S.; GAMA-RODRIGUES, E.; GAMA-RODRIGUES, A. C.; MACHADO, R. C.; BALIGAR, V. C.. Relationships between invertebrate communities, litter quality and soil attributes under different cacao agroforestry systems in the south of Bahia, Brazil. Applied Soil Ecology, v.46, n.3, p.347354, 2010. DOI: http://doi.org/10.1016/j.apsoil.2010.10.006

NAIR, P. K. R.. Agroecosystem management in the 21st century: it is time for a paradigm shift. Journal of Tropical Agriculture, v.46, p.1-12, 2008.

NUNES, L. A. P. L.; ARAÚJO FILHO, J. A.; MENEZES, R. I. Q.. Diversidade da fauna edáfica em solos submetidos a diferentes sistemas de manejo no semiárido nordestino. Scientia Agrária, v.25, n.01, 2009.

NUNES, L. A. P. L.; ARAÚJO FILHO, J. A.; MENEZES, R. I. Q.. Recolonização da fauna edáfica em áreas de Caatinga submetidas a queimadas. Revista Caatinga, Mossoró, v.21, n.3, p.214-220, 2008.

PAUDEL, B. R.; UDAWATTA, R. P.; ANDERSON, S. H.. Agroforestry and grass buffer effects on soil quality parameters for grazed pasture and row-crop systems. Applied Soil Ecology, v.48, p.125-132, 2011.

ROGER-ESTRADE, J.; ANGER, C.; BERTRAND, M.; RICHARD, G.. Tillage and soil ecology: partners for sustainable agriculture. Soil and Tillage Research, v.11, n.1, p.33-40, 2010.

SILVA, J.; JUCKKSCH, I.; FERES, C. I. M. A.; TAVARES, R.. Fauna do solo em sistemas de manejo com café. Journal of Biotechnology and Biodiversity, Gurupí, v.3, n.2, p.59-71, 
2012.

VARGAS, A. B.; CHAVES, D. A.; VAL, G. A.; SOUZA, C. G.; FARIAS, R. M.; CARDOZO, C.; MENEZES, C. E. G.. Diversidade de artrópodes da macrofauna edáfica em diferentes usos da terra em Pinheiral, RJ. Acta Scientiae Et Technicae, Rio de Janeiro, v.1, n.2, p.21-27, 2013. DOI: https://doi.org/10.17648/uezo-ast-v1i2.41

VASCONCELOS, H. L.. Formigas do solo nas florestas da
Amazônia: padrões de diversidade e respostas aos distúrbios naturais e antrópicos. In: MOREIRA, F. M. S.; SIQUEIRA, J. O.; BRUSSAARD, L.. Biodiversidade do solo em ecossistemas tropicais. Lavras: UFLA, 2008. p.323-369.

WASTOWSKI, A. D.; ROSA, G. M.. Caracterização dos níveis de elementos químicos em solo, submetido a diferentes sistemas de uso e manejo, utilizando espectrometria de fluorescência de raios-x por energia dispersiva (EDXRF).

Química Nova, v.33, p.1449-1452, 2010.

A CBPC - Companhia Brasileira de Produção Científica (CNPJ: 11.221.422/0001-03) detém os direitos materiais desta publicação. Os direitos referem-se à publicação do trabalho em qualquer parte do mundo, incluindo os direitos às renovaç̃ões, expansões e disseminações da contribuição, bem como outros direitos subsidiários. Todos os trabalhos publicados eletronicamente poderão posteriormente ser publicados em coletâneas impressas sob coordenação da Sustenere Publishing, da Companhia Brasileira de Produção Científica e seus parceiros autorizados. Os (as) autores (as) preservam os direitos autorais, mas não têm permissão para a publicação da contribuição em outro meio, impresso ou digital, em português ou em tradução. 